\title{
Relationship between magnetic field evolution and major flare event on July 14, 2000
}

\begin{abstract}
Y. Liu and H. Zhang
Beijing Astronomical Observatory, National Astronomical Observatories, Chinese Academy of Sciences, Beijing 100012, PR China

Received 9 February 2001 / Accepted 11 April 2001

Abstract. On July 14, 2000 the active region NOAA 9077 produced one of the largest solar flares (3B/X5.7) seen in recent years associated with a violent halo coronal mass ejection (CME). It had a $\beta \gamma \delta$ magnetic classification and the morphology of the sunspot group and magnetic field changed obviously every day. We present here the relationship between the large scale motions of the spots and the major flare on July 14, based on precise measurements of the proper motions. We find that: (1) The special magnetic morphology and quick, successive fragmentation caused the active region to be always in a high shear configuration; (2) There is a good spatial correspondence between the direction of the movement of one spotgroup and the place where the filament was cut off and activated; (3) The motion characteristics of the rapidly emerging flux system showed a good correlation between spot motions and the largest flares, suggesting that the initiation of the two-ribbon flare on July 14 was promoted by the successive emergence of the flux systems. The intensive major flare was always connected strongly with a newly emerging magnetic flux system (Kálmán 1997). This confirms that $\delta$-configurations and dynamical processes are important in large flares.
\end{abstract}

Key words. Sun: magnetic fields - Sun: activity - Sun: flares - Sun: sunspots

\section{Introduction}

Künzel (1960) pointed out a clear connection between flare productivity and magnetic structure, and the $\delta$ configuration, in which sunspot umbrae of opposite magnetic polarity appear close together within the same penumbra, is a critical ingredient of the solar flare problem. Warwick (1966) confirmed that a large proportion of all major flare events started with the $\delta$ configuration. The characteristics of flare-productive sunspot groups have been studied widely since then (Zirin \& Tanaka 1973; Hagyard et al. 1984; Zirin \& Liggett 1987; Tanaka 1991; Schmieder et al. 1994; Li et al. 1999). The sheared configuration of a magnetic field and $\delta$-type configuration of sunspot groups are essential for strong flare activities. Recently, Sammis et al. (2000), based on 8 years of active region (AR) observations, confirmed that almost all substantial flares occur in $\beta \gamma \delta$ regions.

Why do the sunspot groups in such structures have a higher flare productivity? In this paper, we study the proper motion of sunspots and magnetograms with high spatial and temporal resolution to answer this question.

On July 14, 2000, a large, complex $\beta \gamma \delta$ sunspot group NOAA $9077\left(\mathrm{~N} 17^{\circ}\right)$, produced one of the largest solar

Send offprint requests to: Y. Liu,

e-mail: lyu@sun10.bao.ac.cn flares (3B/X5.7) seen in recent years. The flare started at 10:03 UT and reached its maximum at 10:24 UT. The AR allows us to study the evolution of sunspot group morphology, with a $\delta$-type magnetic structure, during its disk passage in July 2000 (Fig. 1).

We introduce the data used here and the morphology of AR 9077 in Sect. 2, then describe the proper motion measurements and show the result of motions in Sect. 3.1. In Sect. 4 we demonstrate evolution of the AR's vector magnetic fields. In Sect. 5 we discuss the peculiarities of the sunspot motions, the trigger of the flare, and present our conclusions.

\section{Data and morphology}

\subsection{Data}

The data used for this study include $\mathrm{H} \beta$ filtergrams, photosphere filtergrams and photospheric vector magnetograms obtained with the Solar Magnetic Field Telescope (SMFT) (Ai \& Hu 1986; Zhang \& Song 1992) installed at Huairou Solar Observing Station (HSOS); White light (WL, $5000 \AA$ ) images from the Transition Region and Coronal Explorer (TRACE) satellite (Handy et al. 1999); and full-disk magnetograms by the Michelson Doppler Imager (MDI) (Scherrer et al. 1995) on board the Solar 


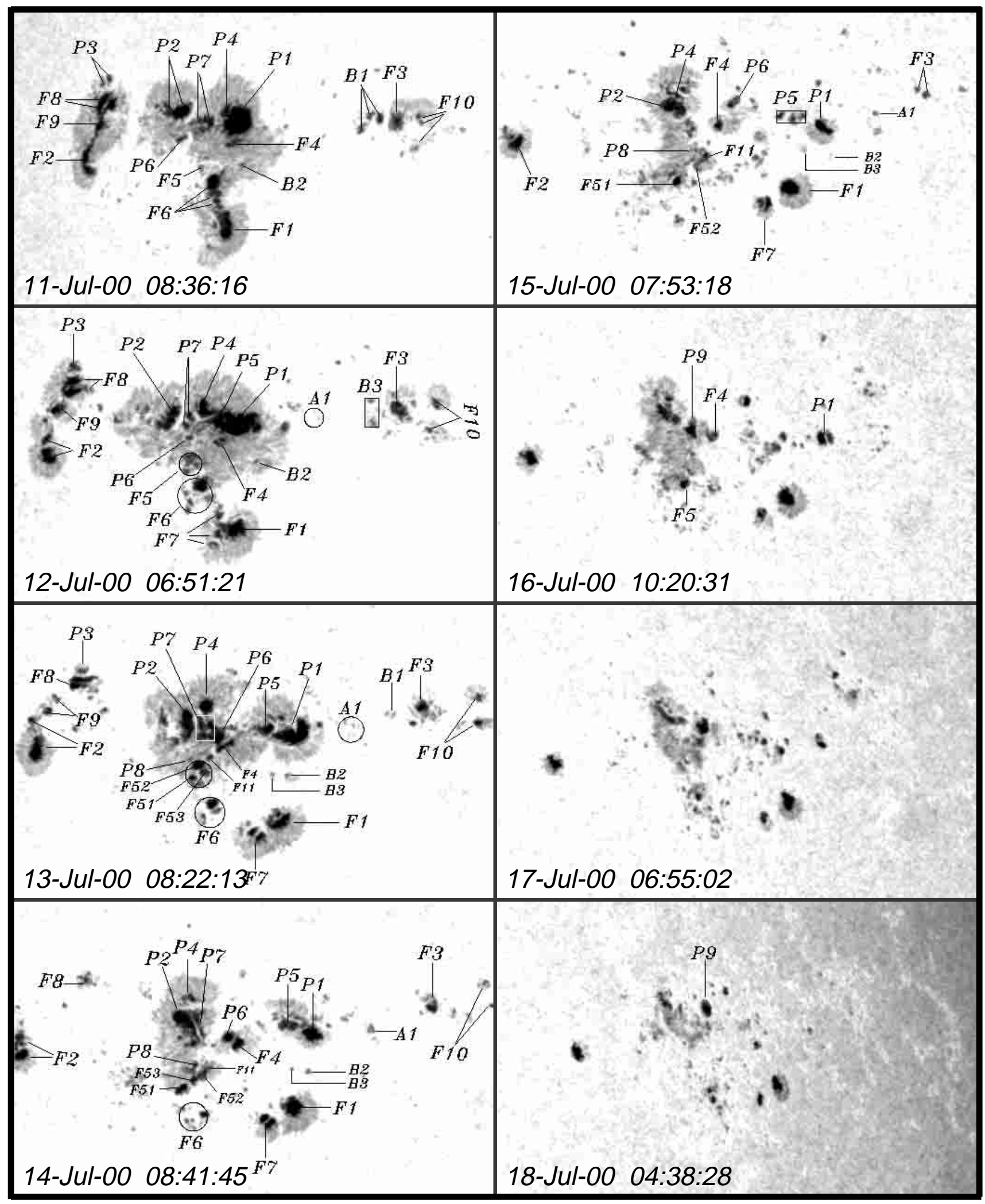

Fig. 1. A time sequence of white-light observations of AR 9077 from TRACE. The size of images is $6^{\prime} \times 4^{\prime}$. The preceding (positive) spots are marked with "P" and "A", following (negative) are marked with " $\mathrm{F}$ " and "B". North is up and east is to the left.

and Heliospheric Observatory (SOHO) (Domingo et al. 1995).
MDI offers us high temporal resolution full-disk data with an interval of $96 \mathrm{~min}$. Its spatial resolution is $1.96^{\prime \prime}$. 
The white light images were flat-field corrected with an $8.5^{\prime} \times 8.5^{\prime}$ field of view (FOV), 1 arcsec spatial resolution and about 2 hours temporary resolution. Combined with the full-disk, high resolution MDI data, local magnetograms from HSOS and the corresponding WL images, we can easily identify magnetic polarity and position of a spot, and calculate its motion precisely.

\subsection{Morphology}

AR 9077 first appeared east of the Sun on July 7 with an obvious $\delta$-type magnetic structure and a distribution of polarities which violated the Hale-Nicholson rule. The positive flux was emerging fast from the northeast and north toward the main negative polarity. Figure 2 shows the evolution of the sunspot group during July 11-18 without projection correction as the AR was not far from the disk central meridian. The largest of the group was about 15 degrees in longitude and 5 degrees in latitude. We describe the spots by their polarities with "P", "A" and "F", "B" representing preceding (positive) and following (negative) magnetic polarity respectively. Sunspot P1 matured after emerging from below the photosphere on July 11. Its penumbra was shared with $\mathrm{F} 6$, forming a $\delta$ type magnetic structure. The spot F4 was near the magnetic neutral line of the $\delta$ configuration.

On July 12, P1 elongated to the east-west, and some small parts of its umbra, P4 and P5, moved away from the east end of P1. We think that it is due to P1's fast acceleration forward (westward) that the two parts were separated from P1. On July 13, P5 still followed P1 at its east end, while $\mathrm{P} 4$ moved into the penumbra northwest of P2. P1 became smaller and tiny satellite spots formed around it. P1 continued to shrink and disappeared by July 18 .

From Fig. 1 we can see that sunspot P2 rotated anticlockwise during the first four days, which suggests that the flux system of P2 which emerged from below was driven by a twisting motion. We found that during the first period $\mathrm{P} 2$ rotated with a high rotating velocity of about $20^{\circ}$ day $^{-1}$. However, during the next period it rotated little.

The negative spots F1, F5 and F6 changed their alignment direction from north-south to east-west rapidly between July 11-14, indicating different motion patterns among them. F5 was a new emerging flux at the polarity inversion line (PIL) of the $\beta \gamma \delta$ configuration on July 11; it developed into a small spot group in P2's penumbra (F51, F52 and F53, forming a triangle) which is marked by a circle in the image of July 13, when another positive spot, $\mathrm{P} 8$, formed almost at the same position. The PIL crossed between P8 and F5. On July 14, the components of F5 were in a straight line along the PIL, and F53 disappeared after the major flare, leaving the other two components weakened there.

Spot P7 was a group of many tiny spots formed in the penumbra of P1, they grew slowly and merged with P2's

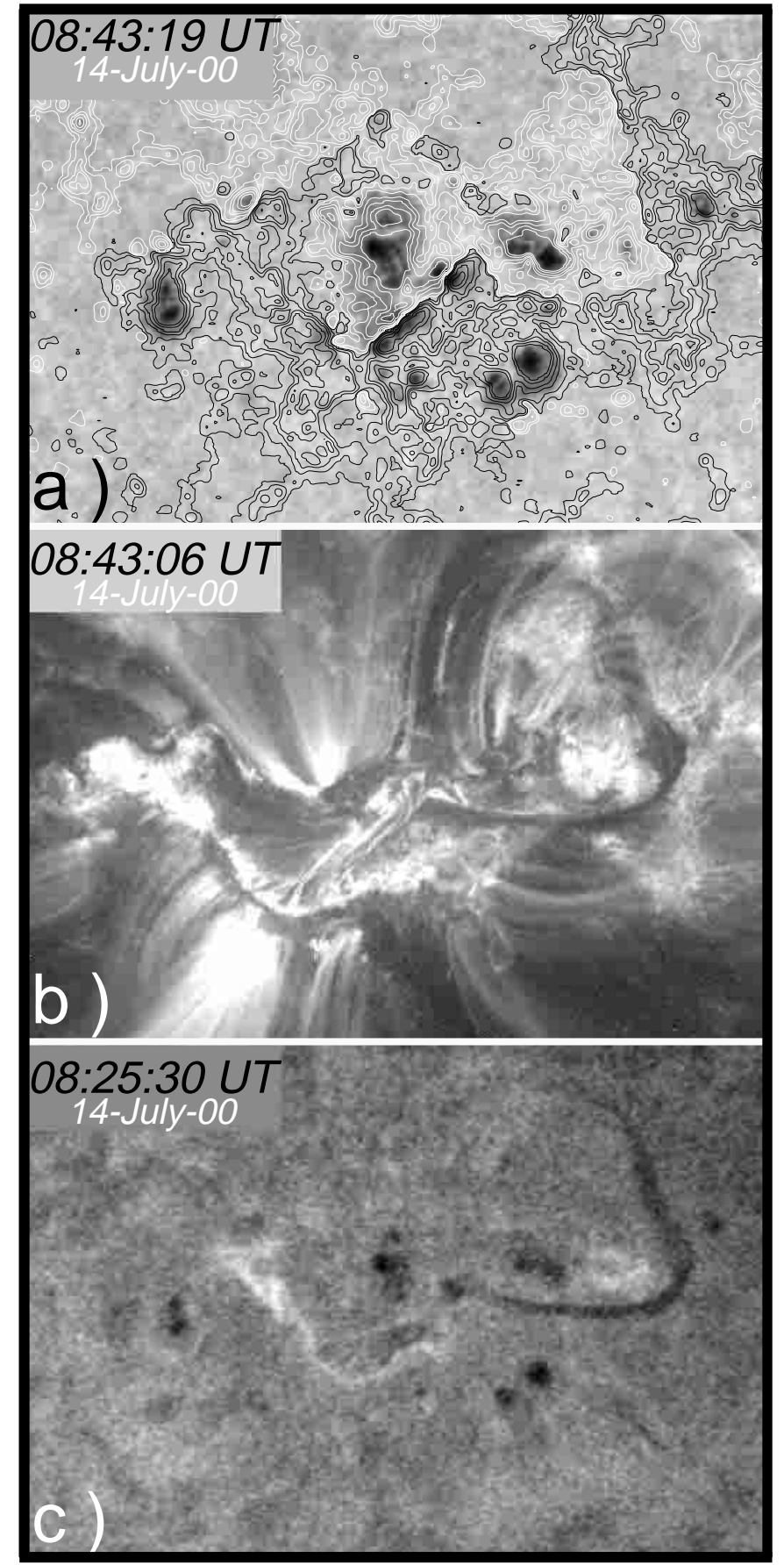

Fig. 2. Global morphology of the AR 9077 on July 14, 2000. a) A white-light image overlaid by the longitudinal photospheric magnetic field of HSOS, showing the polarity of every spot clearly. White contours represent positive polarity, black represent negative; b) A TRACE $171 \AA$ image of the region at the same time, notice a twisted filament formed along the right $\mathrm{PIL}$; ) An $\mathrm{H} \beta$ image taken from HSOS. The FOV is $4.9^{\prime} \times 3.3^{\prime}$.

penumbra on July 13. On the next day P7 turned into a slender line very close to the umbra of P2.

The negative umbrae F8, F9 and F2 were in an integrated penumbra, with a small positive spot P3 in its north, forming a $\delta$ configuration. Another small negative 


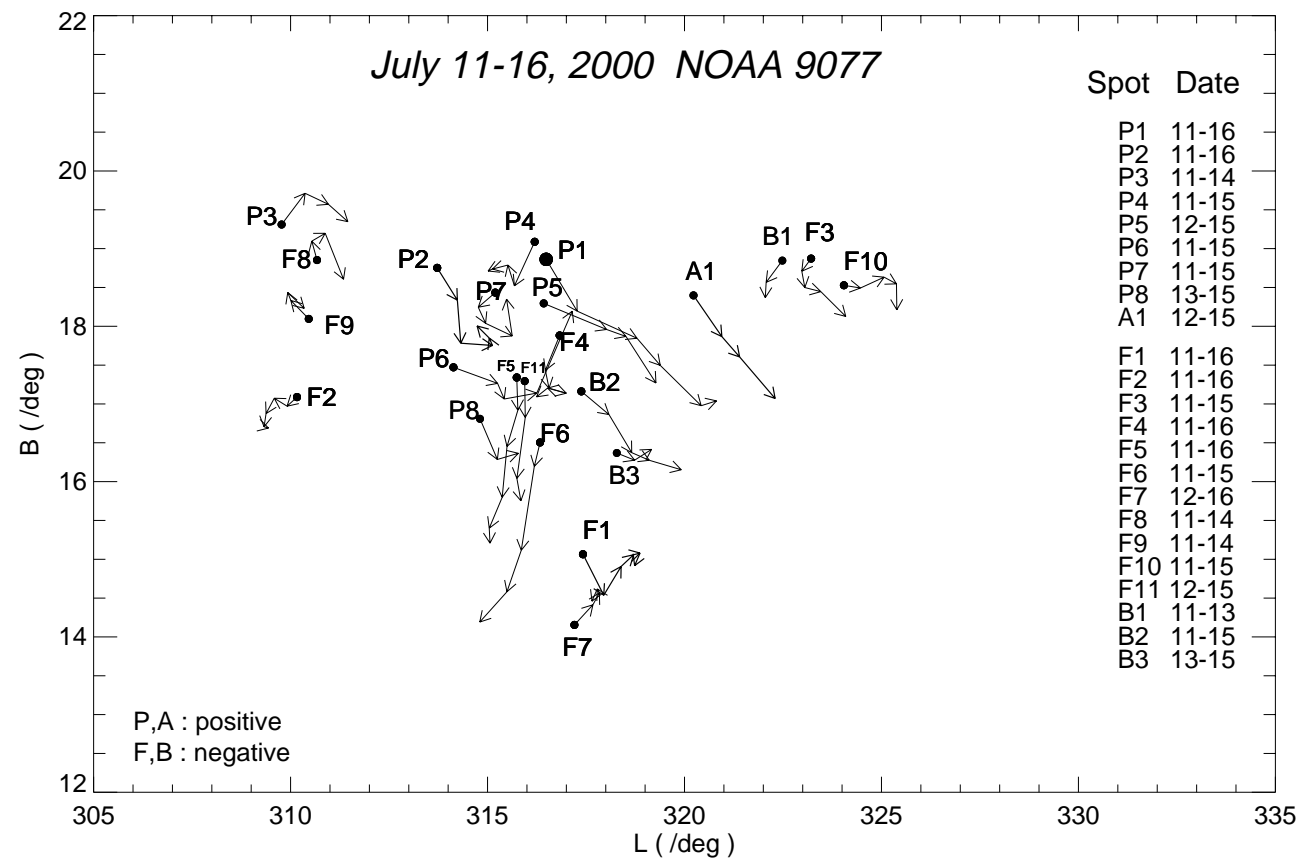

Fig. 3. Proper motions of the spots referenced in Fig. 1. The arrowheads and black points represent the positions at 12:00 UT, the coordinates are in the Carrington system.

spot group (F3 and F10) lay west of the main group of $\mathrm{P} 1, \mathrm{P} 2$ and $\mathrm{F} 1$.

Thus, before the AR moved to the central meridian on July 14, important evolution of the sunspot group had occurred. About 130 flares of $\mathrm{H} \alpha$ and soft X-ray (SXR) occurred during its disk passage on the Sun (cited from the SEC site), and 3 of them were of X-class in SXR, of which the most prominent was the X5.7 two-ribbon flare on July 14, 2000 (the other two SXR flares were an X1 flare on July 11 and an X1.9 on 12). Figure 2 presents the global morphology of the AR just before the flare on this day. The top image is a white-light one overlaid by the longitudinal photospheric magnetic field of HSOS, showing the polarity of each spot clearly; The middle is the corresponding TRACE $171 \AA$ image, where a twisted filament is seen formed along the right PIL; The lower one is an $\mathrm{H} \beta$ image taken from HSOS. Images from SOHO/LASCO spacecraft showed that a large, fast-moving halo CME followed the major flare of July 14 and headed earthward.

\section{Proper motion measurement and results}

\subsection{Proper motion measurement}

In order to obtain the heliospheric position of each spot, we utilize the full-disk MDI's magnetograms, whose data array is $1024 \times 1024$, the same as the WL images of TRACE. The MDI data are translated into the threedimension (3-D) heliospheric coordinate system by a standard IDL procedure. To trace individual sunspot umbra over several days, we should take into account the effects of solar differential rotation, the $B$-angle of the solar north pole, and the Earth's orbital motion. On July 14, 2000, the $B$-angle was $4.26^{\circ}$ toward the Earth, and the angle changed about $0.1^{\circ} \mathrm{day}^{-1}$. According to Zirin (1989), the Earth's orbital velocity is $0.9865^{\circ}$ day $^{-1}$. The differential rotation is corrected by the method of Newton \& Num (1951) for low latitude $\left(\mathrm{N} 17^{\circ}\right)$. The data reduction procedure is summarized as following:

1. Identify each spot's magnetic polarity from magnetograms of HSOS and WL images of HSOS and TRACE (Fig. 2a). Translate the MDI images into 3-D global coordinate system, with $B$-angle correction;

2. Contour MDI's local region of interest, locate a spot's position on the MDI full-disk image, and calculate the spot's present heliospherical coordinates;

3. After removing the effect of the solar differential rotation and the Earth's orbital rotation, we obtain the spot's new position at 12:00:00 UT on July 14, when the spot group was at the central meridian $\left(L_{0}\right.$ was $315^{\circ}$ in Carrington system);

4. Iterating (1), (2), (3) we determine the positions of the spot over several days and plot them in the Carrington coordinate system (Fig. 3).

As we analyse only one WL image each day, the motion velocity calculated is an average effect of 24 hours (86 $400 \mathrm{~s}$ ), demonstrating proper motion on a long time scale.

\subsection{Results}

To identify the connection between proper motions and the major flare of July 14, we measure the motions from July 11 to 16. 


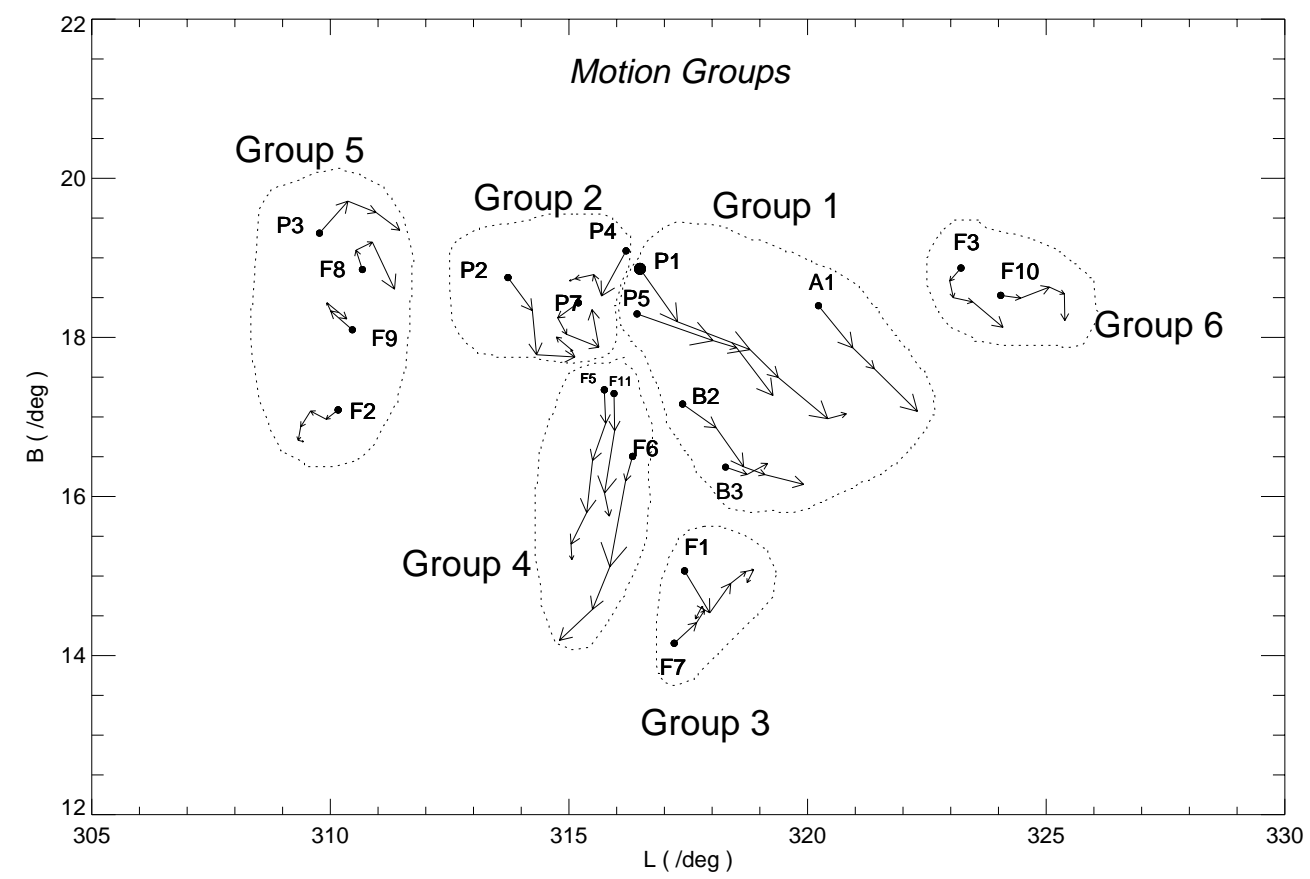

Fig. 4. Motion groups from Fig. 3, contours indicate groups of spots which have a similar displacement. The size of the arrowhead is proportional to the displacement.

\subsubsection{Global proper motions}

Trajectories of the persistent umbrae are very complicated. Spots named "A" and "B" are small and shortlived but contributed to the magnetic topology changes and shear enhancement along the PIL.

We find there are six groups of spots with respective motion patterns (Fig. 4). Note that P6 and P8, not shown in Fig. 4, belong to group 2, and F4 belongs to group 4 .

Group 1 was moving forward while group 2 rotated anti-clockwise. Group 4 moved directly southward and the PIL seemed to be thereby stretched toward the equator.

\subsubsection{Motion of group one}

One of the dominant motions in the AR was that of group 1, moving southwest and including preceding and following spots. It's generally thought that the "HaleNicholson force" could drive the preceding spots westward, but why did the following spots B2 and B3 also move west? Wang et al. (1991) proposed a general source other than that which produces the Hale-Nicholson polarity orientation.

We examine group 1 to determine how the motions progressed. The kinetic energy built up due to shear motion is large enough to power large flares (Krall et al. 1982; Tanaka 1991; Wang et al. 1991), but we are surprised that a large flare broke out when the motion was small. However, spots of group 1 show a good correspondence between spot motion and the largest flares.

Figure 5 shows the variety of spot proper motion velocities versus date. A1, B2, B3 and P5 were short-lived relative to spot P1, they disappeared on July 15. From this figure, we can see four peculiarities:

1. Almost every spot except B3 accelerated from July 13 to July 14. Varying from $7.5 \times 10^{-7} \mathrm{~km} \mathrm{~s}^{-2}$ for P1, $8.0 \times 10^{-7} \mathrm{~km} \mathrm{~s}^{-2}$ for P5 and A1, $6.0 \times 10^{-7} \mathrm{~km} \mathrm{~s}^{-2}$ for B2, they are comparable to that of another super AR 5395 (Wang et al. 1991). If umbrae are the intersections of the flux loops deep in the convection region with the photosphere, such high acceleration reflects rapid emergence of flux loops, implying the complexity of the original magnetic topology and instability;

2. Almost all of the spots of the group had a lower velocity $\left(0.05-0.10 \mathrm{~km} \mathrm{~s}^{-1}\right)$ on July 13 , when no X-class flare took place. On the other days (July 11, 12 and 14) P1 and P5 reached high velocities $\left(0.20-0.25 \mathrm{~km} \mathrm{~s}^{-1}\right)$ on July 12 , A1 and B2 reached $\left(0.10-0.15 \mathrm{~km} \mathrm{~s}^{-1}\right)$;

3. P1's two peaks of velocity corresponded to the two largest X-class flares (X1.9 on July 12, X5.7 on July $14)$, and the third high velocity $\left(0.14 \mathrm{~km} \mathrm{~s}^{-1}\right)$ appeared on the day the third largest X-class flare took place;

4. After the major flare of July 14, P5, A1, B2 and B3 disappeared, and P1 slowed from $0.21 \mathrm{~km} \mathrm{~s}^{-1}$ to $0.06 \mathrm{~km} \mathrm{~s}^{-1}$. From then on there were no large $\mathrm{X}$ class flares.

The good relationship between large flares and the motion of umbrae is an evidence that the motion of the spot, i.e., emerging of flux, is a necessary condition to produce large flares. Thus, we can assess the probability of a large flare from the abnormal acceleration of spots.

To test this, Fig. 6 shows the process of the major flare on July 14 . The left column are $\mathrm{H} \beta$ filtergrams, the right are EUV images from TRACE. On the chromosphere, we 

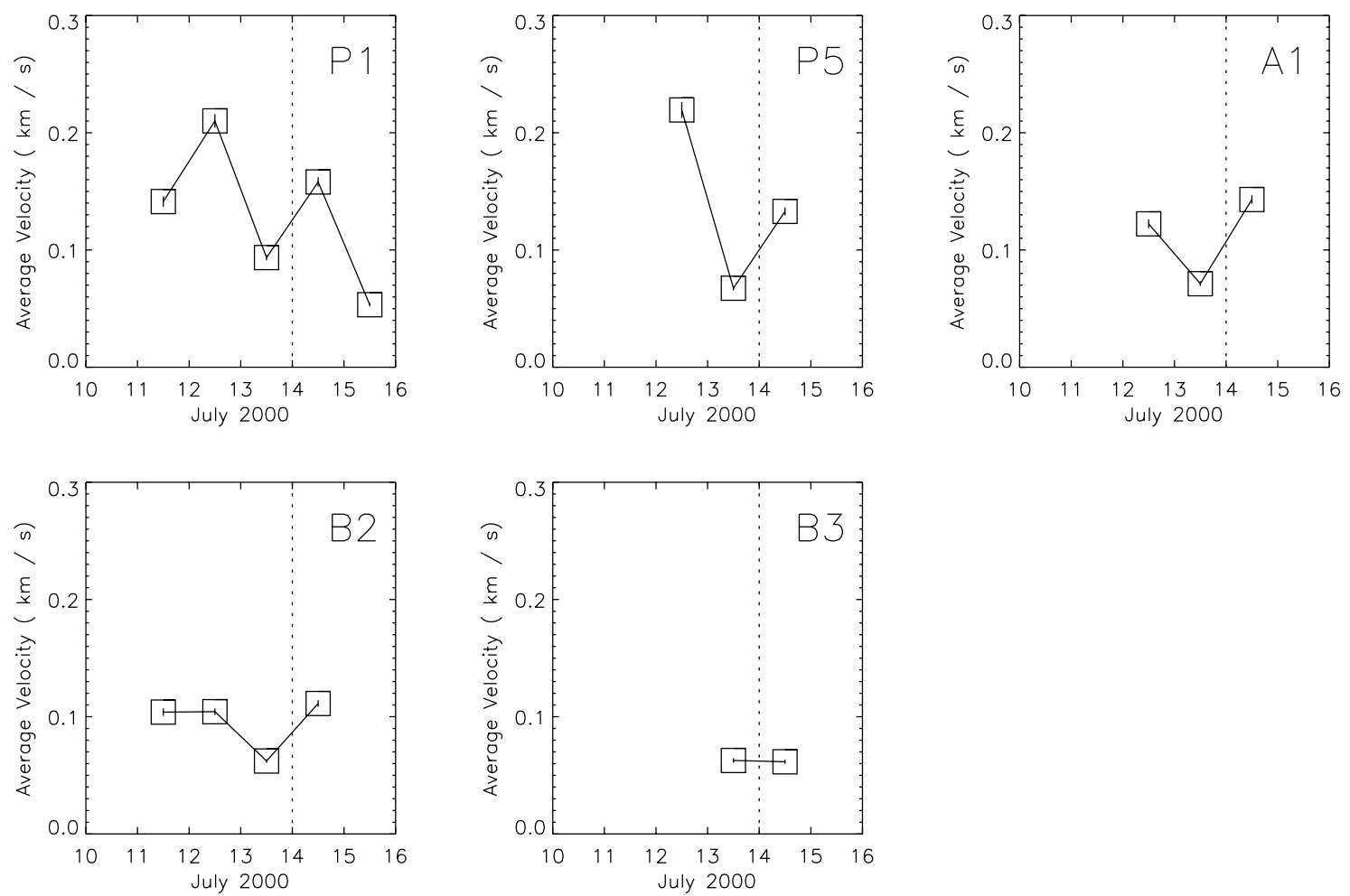

Fig. 5. Velocity of spots from group one as a function of date. Each point is represented by a square, the errors are indicated by a black vertical bar in the squares.

see a left-inclining " $\omega$ " shape filament, with its inflected point "D" in the direction of the motion of group 1. At 09:48 UT, the filament cut off and the south part seemed to be activated and rise up, suggesting the PIL was experiencing significant dis-equilebrium. At 10:10 UT a surge erupted from point " $\mathrm{D}$ ", and the $\mathrm{H} \beta$ flare kernel of the main event appeared nearby. After the surge, a two-ribbon flare developed rapidly along the PIL at 10:19 UT. The same scenario can be seen in the synchronous observations of TRACE in the right column of Fig. 6.

\subsubsection{Motions of spots along the PIL}

The PIL of the AR 9077 was sinuous, we draw the main part of it in Fig. 7. We re-mark the point "D" of Fig. 6 as the inflection point in this figure. Point " $\mathrm{D}$ " was headed by the motion vector of group one.

Small circles in Fig. 7 represent positions of spots on July 14. Big umbrae except P1 and P5 seemed stable relative to the others and caused little influence on the PIL. The presence of the other spots altered the position of PIL, which can be seen from the magnetograms in the next section. The west broadening was brought about by P6 and P8. P6 turned northwest when it met F4, a small spot that paced back and forth near the apex of the mid PIL. P6's northward movement kept the total PIL in a highly " $\omega$ " shape.

Right part of the PIL, appeared to be pushed forward through the negative field region by the preceding spots of group 1. B2 and B3, moved to the left, and B1 to the right.
Together they increased the gradient of the magnetic field across the PIL.

The quick fragmentation of the sunspot group AR 9077 lead to a continuous restructuring of the overall magnetic filed pattern, and the PIL was kept in a state of disequilibrium. It was the motion of group 1 that promoted the eruption of the major flare event.

\section{Evolution of magnetic field}

\subsection{Large scale evolution of vector magnetic field}

The AR 9077 was in a $\beta \gamma \delta$-configuration with a magnetic field of medium intensity, but high stress and shear along the PIL was maintained and even strengthened by the motion of spots. In the following, we describe the mechanism further.

Figure 8 shows the large scale evolution of the vector magnetic field of AR 9077. The isocontours, overlying the grey magnetograms, represent longitudinal components with solid lines showing positive polarity and dash lines, negative polarity. The contour levels are 160, 424, 677, 1071 G. The bars are transverse components with their length proportional to the intensity. Some parts of the PIL are observed to have steep gradients across them (such as boxes "A", "B" in Fig. 8). The morphologic changes of the field correspond well to the motions of spots. 


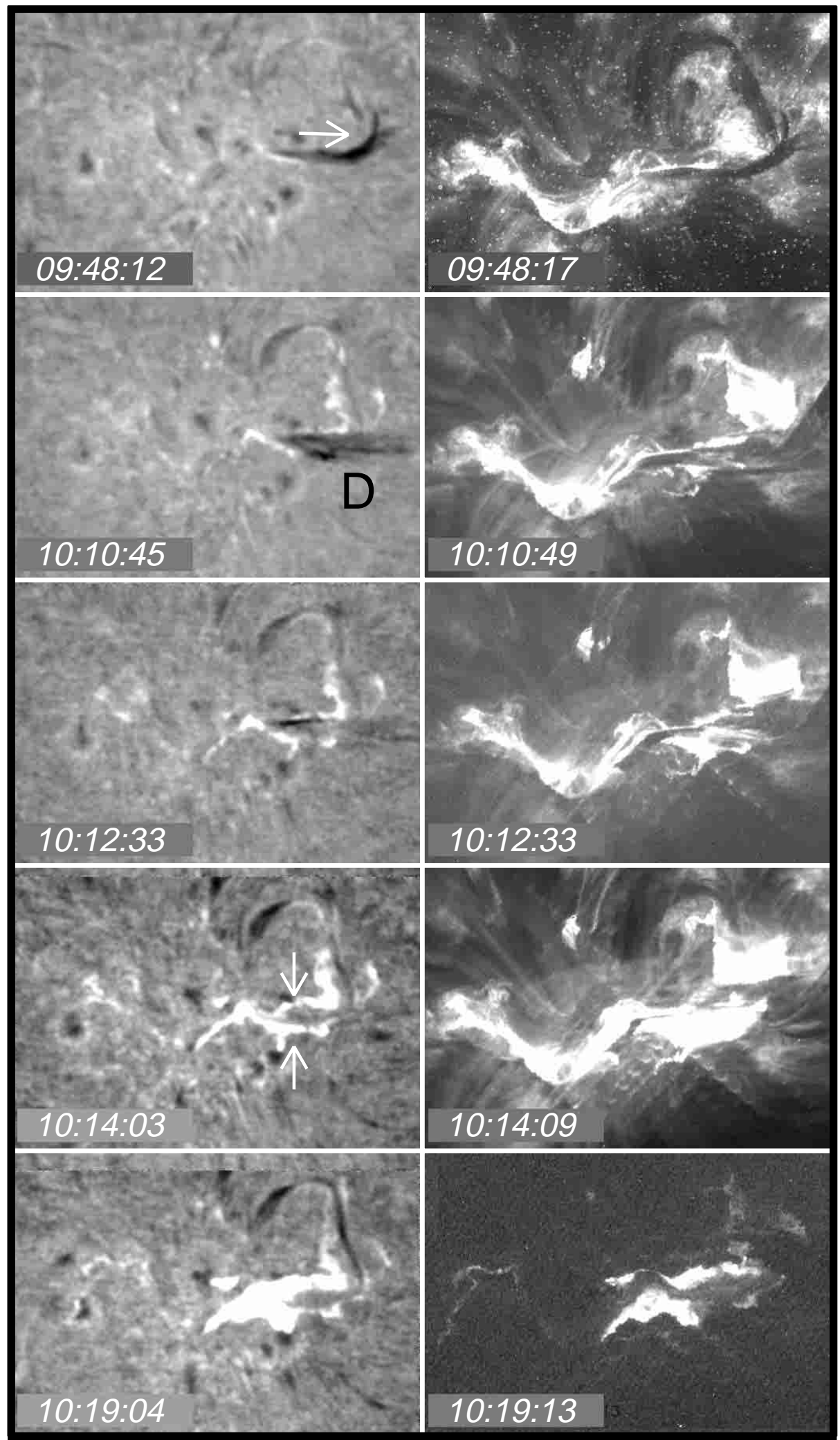

Fig. 6. A series of $\mathrm{H} \beta$ (left column) and TRACE images (right column, with $195 \AA$ at 10:12:33, others are in $171 \AA$ ), obtained almost at the same time, which show the quick changes near the point "D" before and during the major flare took place on July 14 . The horizontal arrow indicates the activation near the inflection point of the filament, and the two vertical arrows indicate the two ribbons of the major flare. The FOV is $5.2^{\prime} \times 3.6^{\prime}$.

\subsubsection{Magnetic shear in Box "A"}

On July 11 , the $\delta$-type magnetic structure was very compact. The penumbra of positive umbrae P1 and P2 was shared with a negative field and the transverse components in box "A" were almost parallel to the neutral line, i.e., the shear angle was very large $\left(80^{\circ}-90^{\circ}\right.$, by the method of Teuber et al. 1977), suggesting much nonpotential energy was stored along the PIL.

The spot P6 was a positive island connecting with its eastern negative elements. Its mixture into P1 and P2 on July 12 made the PILs in box "A" and "B" longer and 


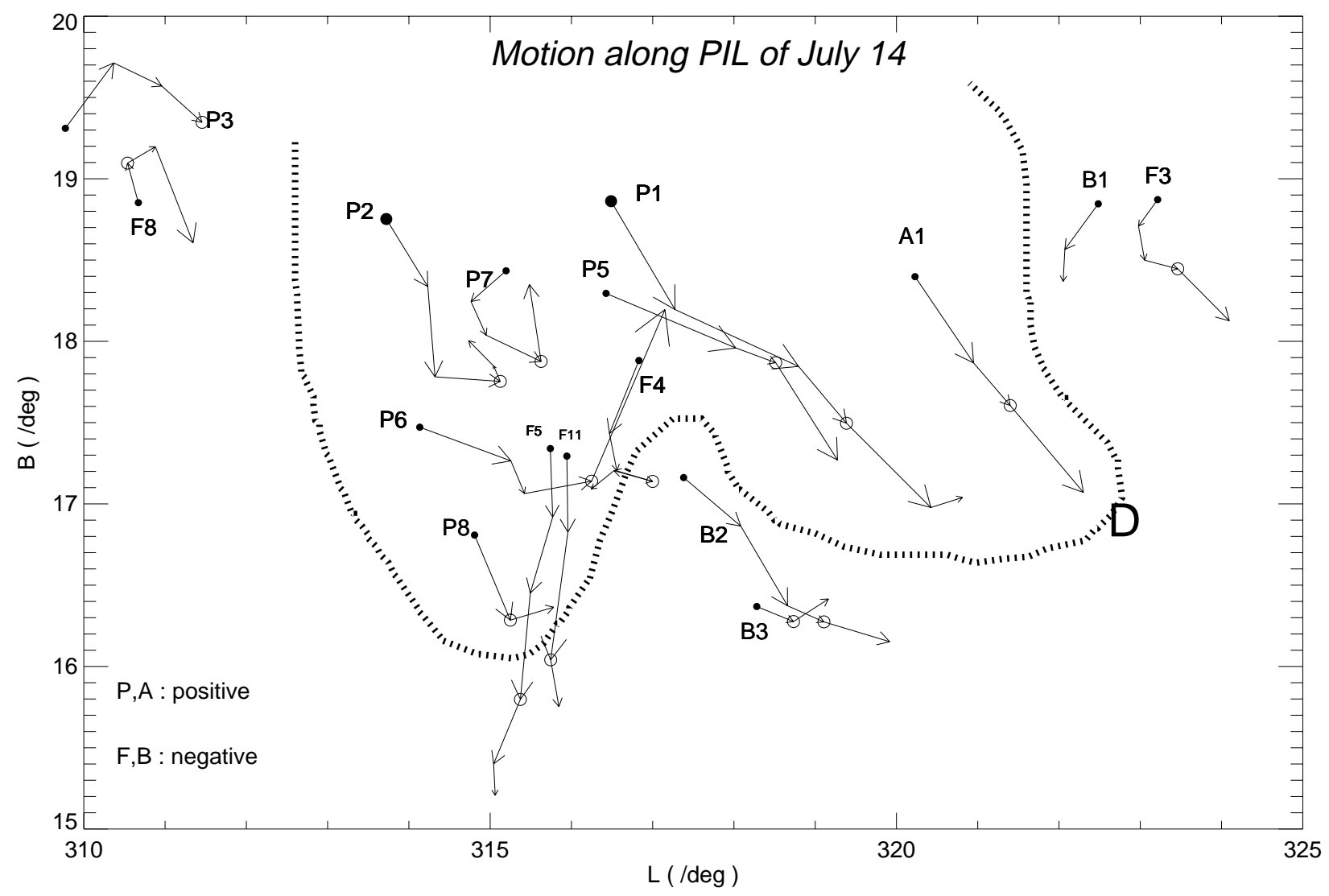

Fig. 7. Motions along the PIL of July 14, indicated by a thick dash line. "D" is the point of the PIL's inflection, as in Fig. 6, circles are positions of spots on July 14, and the size of the arrowhead is proportional to the displacement.

steeper (see following subsection). P7 emerged between P1 and P2, whose transverse component didn't cross the PIL. The negative spot F4 formed south of the PIL in "A".

On July 13, P6 had intruded between P7 and F4, and formed a new sheared pair of polarities on both sides of the PIL. Behind P6, a new spot, P8, formed in the penumbra east of "A", shifted southward, and merged with F5.

Spot F5 was divided into three smaller ones (F51, F52 and F53), in a line at the south side of the PIL on July 14. As the distance between F5 and P8 was shortened by their relative motion, the magnetic gradient across the PIL became steeper. Two small positive fluxes E1 and E2 emerged from the negative field.

On July 15, the rapid relative motion between F4 and P6 became prominent (Fig. 7). Even after the major flare on July 14 the high shear state in box "A"' seemed little changed.

\subsubsection{Magnetic shear in Box "B"}

The PIL in box "B" is a subsetion of the entire PIL, but its evolution is representative of the larger structure. P2 was connected with the negative field north and east of it (regions S2, S3), and the transverse component in box "B" deviated slightly on July 11. As P6 moved and merged into $\mathrm{P} 1$ and $\mathrm{P} 2$, sunspot $\mathrm{P} 2$ rotated around its own axis, forming a seam south of $\mathrm{P} 2$ with its $B_{\perp}$ components parallel to it (box "B" on July 12 in Fig. 8). Even after the flare, no obvious reconnection was seen in box "B" from the tranverse field analysis of July 15.

\subsection{Quick evolution and emerging fluxes}

Some local regions underwent rapid evolution of their original fields. The most marked change along the longitudinal field was the formation of a tongue into the positive field. The edge of the tongue was translated from the lineshaped PIL on July 11 into a " $\wedge$ " shape on July 14; the peak of it was named point "I" plotted in Fig. 8. The edge changed into a " $\cap$ " shaped tongue. Indeed, the edge of the tongue was the PIL discussed in box "A". For group 1, the border pushed its western polarities into a more compact configuration, as it had to squeeze close to the southwestern negative polarity field. On July 14, two anti-polarity fluxes "E1" and "E2" had emerged in the negative field to the south of the frontier. They canceled with the negative polarity and merged with the frontier, forming two peninsulae crossing the PIL. Another important flux formed at "D" in Fig. 8. Additionally, many small moving magnetic features (MMFs) appeared from the north of P1 and P2; they are always in pairs of mixed polarities. There were also considerable MMFs that were 


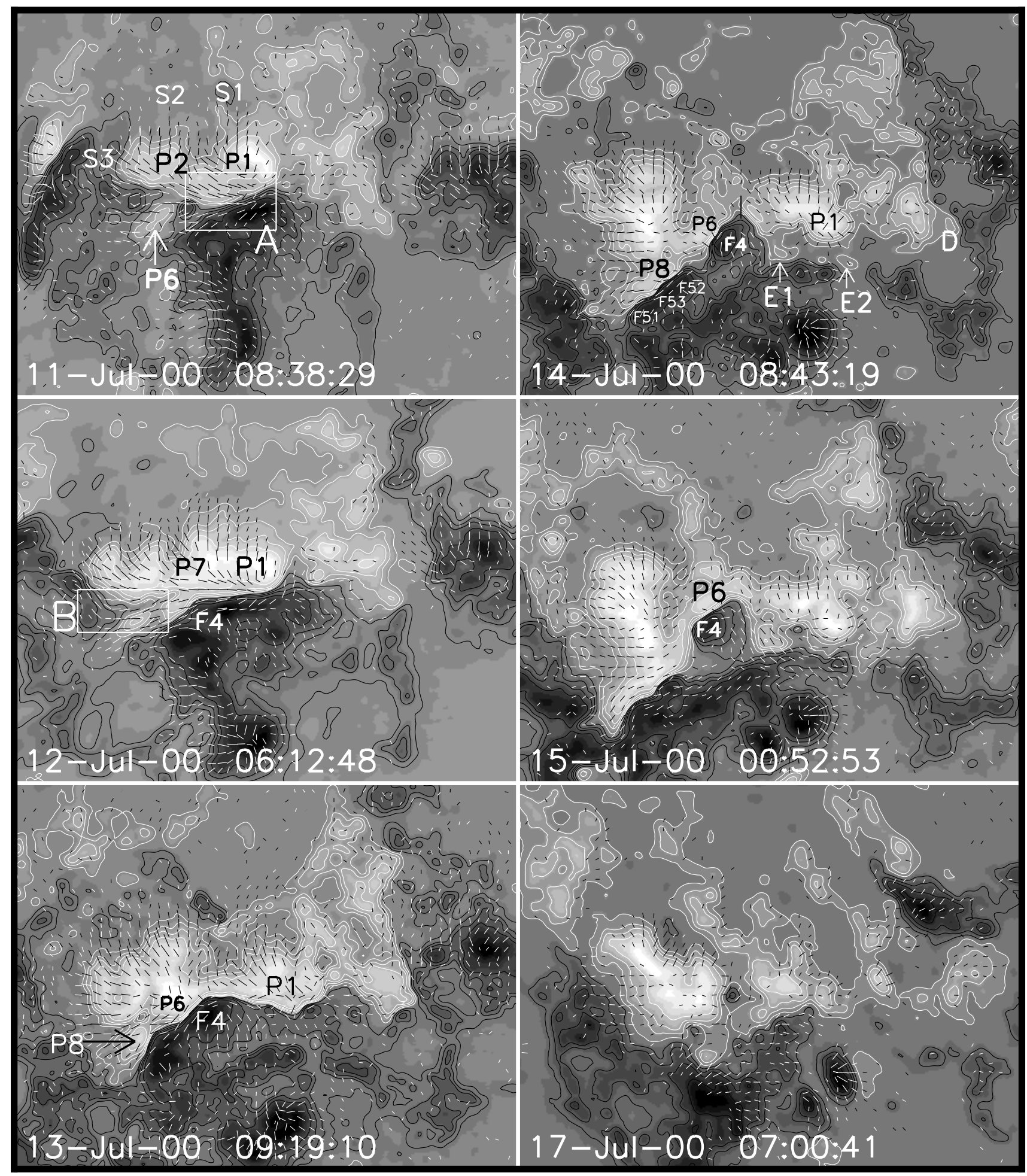

Fig. 8. A time sequence of the vector magnetograms from July 11 to 17 without 16 . The FOV is $3^{\prime} \times 2.3^{\prime}$. The contour levels of the longitudinal field are 160, 424, 677, 1071 Gauss. White contous are negative polarity and black are positive. Point "D" is the place of initiation of the major flare. E1 and E2 are two new emerging fluxes on July 14. Notice the evolution of a high shear configuration along the PIL and high magnetic gradient across it.

only positive, which are attributed to the decay of the AR (Zhang \& Ai 1992).

Zhang et al. (2001) examined in detail the magnetic evolution in the AR 9077 on July 14, just before the major flare event. Here we introduce some other information on the evolution of the magnetic field before and after the major flare (maximum at 10:24 UT, 14 July).

The filament terminated one of its ends at the tip of the tongue, where P6 and F4 cancelled, Fig. 9 shows the evolution of the shear along the PIL between P6 and F4. 


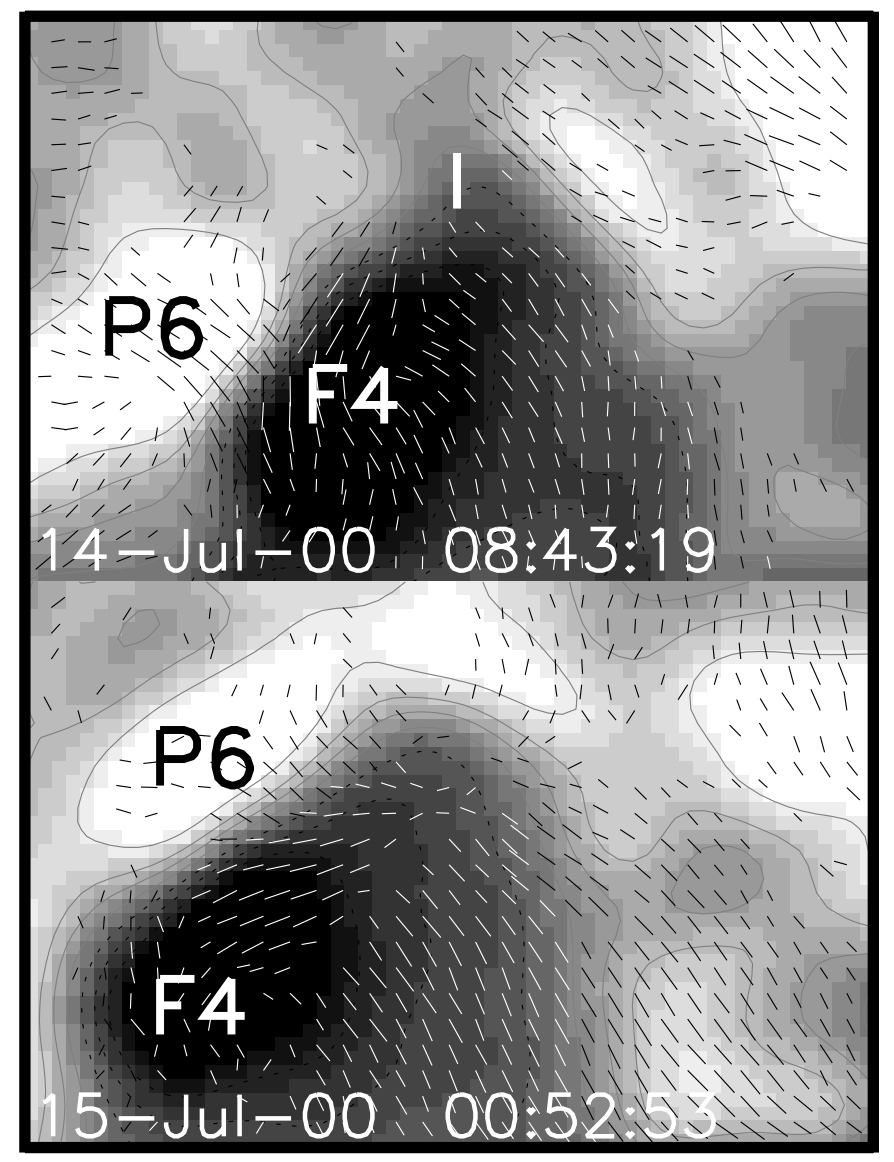

Fig. 9. HSOS vector magnetograms of a local region in the AR 9077 on July 14 and 15; the FOV is limited as a region of $0.6^{\prime} \times 0.4^{\prime}$ near the point "I" marked in Fig. 8, July 14 .

We notice that after the flare event, which had released large nonpotential energy, the PIL between P6 and F4 still maintained its original high shear. This is because the spot $\mathrm{P} 6$ moved at a high speed of $0.20 \mathrm{~km} \mathrm{~s}^{-1}$ northwestward along the PIL and F4 moved back, as mentioned in Sect. 3.2.3. We conclude from this that the rapid emergence of a flux system is one important source of the formation and maintenance of shear.

\section{Discussion and conclusion}

The magnetic field of AR 9077, which is one of the most flare-productive regions in the present $23 \mathrm{~d}$ sunspot cycle, was in a typical $\beta \gamma \delta$ class. It produced nearly 130 flares, including $3 \mathrm{X}$-class flares, one of which was the largest (3B/5.7X, associated with a halo CME and an eruption of a filament) seen in recent years. We have examined this AR's motion characteristics in detail and found (1) The special magnetic morphology and quick, successive fragmentation forced the active region to remain in a high shear configuration before and after the major flare event of July 14; (2) There's a good spatial correspondence between the general direction of movement of one spotgroup (group one in Fig. 4) and the place where the filament was cut off and activated; (3) The motion characteristics of the rapidly emerging flux system showed a good correlation between spot motions and the largest flares, suggesting that the initiation of the two-ribbon flare on July 14 was promoted by the successive emergence of the flux systems.

The intensive major flare was connected to the newly emerging magnetic flux system (Kálmán 1997). The AR confirms that $\delta$-configuration and dynamical processes are crucial for large flares.

The questions of why a region like AR 9077 was in such motion and of what the driving force might be, are a major focus of solar physics. If we image the motion of sunspots as a geometric motion of the intersecting parts of an emerging magnetic flux loop with the photosphere, the topological structure of the fluxtube system is important to understanding particular patterns of proper motion of a sunspot group. Ishii et al. $(1998,2000)$ suggested that the flare occurrence depends on the formation process of the $\delta$-type configuration or the magnetic shear. Based on the precise measurements of two ARs (NOAA 4201 and NOAA 5395), she concluded that the twisted structure of the emerging flux bundle should be the key ingredient for the high flare-productivity of a sunspot group. The scale of the twisted flux bundle as well as the strength of the twist could determine the flare-activity level. $\beta \gamma \delta$ is one of the most complex magnetic field structure, and the spatial topology should also be very complicated. The motions of spots cause the footpoints of flux system through the photosphere to shift, which will enhance the magnetic shear. When the original magnetic configuration is deformed, current sheets can be created at the surfaces that divide different magnetic flux systems, and the reconnection will release the magnetic free energy.

The construction of a topological model of such complex group as AR 9077 is a difficult task. Most axisymmetric models have neglected the prominence prior to the major flare and the CME. In AR 9077, an eruption of an untwisted filament was seen just before the flare (Fig. 6); its activation may have caused some deformation of the magnetic topology. Recently, Amari et al. (2000) argued that the flux tubes should play a crucial role in the theory of large-scale eruptive phenomena such as CME and tworibbon flares, which we should take into account in future work.

Acknowledgements. The work was supported by the National Natural Science Foundation of China, grants no. 19791090 and 10073013. The authors are grateful to staff at Huairou Solar Observing Station for their support the excellent observations. We are indebted to all members of TRACE and SOHO/MDI teams for providing their wondful data. SOHO is a project of international coorporation between ESA and NASA. We are also grateful to the unknown referee for his or her valuable comments that helped to improve this paper. 


\section{References}

Ai, G., \& Hu, Y. 1986, Publ. Beijing Astron. Obs., 8, 1 Amari, T., Luciani, J. F., Mikic, Z., \& Linker, J. 2000, ApJ, 529, L49

Bumba, V., Klvaňa, M., Kálmán, B., \& Györi, L. 1993, A\&A, 276,193

Domingo, V., Fleck, B., \& Poland, A. I. 1995, Solar Phys., 162,1

Hagyard, M. J., Smith, J. B., Jr., Teuber, D., \& West, E. A. 1984, Solar Phys., 91, 115

Handy, B. N., Acton, L. W., Kankelborg, C. C., et al. 1999, Solar Phys., 187, 229

Ishii, T. T., Kurokawa, H., \& Takeuchi, T. T. 1998, ApJ, 499, 898

Ishii, T. T., Kurokawa, H., \& Takeuchi, T. T. 2000, PASJ, 52, 337

Kálmán, B. 1997, A\&A, 327, 779

Krall, K. R., Smith, J. B., Jr., Hagyard, M. J., West, E. A., \& Cummings, N. P. 1982, Solar Phys., 79, 59

Künzel, H. 1960, Astron. Nachr., 285, 271

Li, W., Chik-Yin Lee, Chae, J., et al. 1999, Ninth European
Meeting on Solar Physics: Magnetic Fields and Solar Processes, ed. A. Wilson, ESA SP Ser, 448, 169 Newton, H. W., \& Nunn, M. L. 1951, MNRAS, 111, 413

Sammis, I., Tang, F., \& Zirin, H. 2000, ApJ, 540, 583

Schmieder, B., Hagyard, M. J., Ai G., et al. 1994, Solar Phys., 150, 199

Scherrer, P. H., Bogart, R. S., Bush, R. I., et al. 1995, Solar Phys., 162, 129

Tanaka, K. 1991, Solar Phys., 136, 133

Teuber, D., Tandberg-Hanssen, E., \& Hagyard, M. J. 1977, Solar Phys., 53, 97

Wang, H., Tang, F., \& Zirin, H. 1991, ApJ, 380, 282

Warwick, C. C. 1966, ApJ, 145, 215

Zhang, H., \& Song, M. 1992a, Solar Phys., 138, 69

Zhang, H., \& Ai, G. 1992b, Solar Phys., 140, 307

Zhang, J., Wang, J., Deng, Y., \& Wu, D. 2001, ApJ, 548, L99

Zirin, H., Astrophysics of the Sun (Cambridge: Cambridge Univ. Press)

Zirin, H., \& Liggett, M. A. 1987, Solar Phys., 113, 267

Zirin, H., \& Tanaka, K. 1973, Solar Phys., 32, 173 